Journal of Advanced College of Engineering and Management, Vol. 6, 2021

\title{
NUMERICAL SIMULATION STUDIES ON STALL SUPPRESSION OF A NACA0015 AIRFOIL
}

\author{
Biswash Shrestha $^{1}$, Nawraj Bhattarai ${ }^{2}$ \\ ${ }^{1,2}$ Department of Mechanical Engineering, Pulchowk Campus \\ Tribhuvan University, Lalitpur, Nepal.
}

\begin{abstract}
This study aims to achieve an improved airfoil performance at low Reynolds number, and to determine the optimum position and size of rectangular cross-section burst control plate (BCP) to suppress stall in airfoil. The type of airfoil used in the present study is NACA0015 (National Advisory Committee for Aeronautics) airfoil with $200 \mathrm{~mm}$ of chord (c) length. Here, rectangular cross-section burst control plates with different sizes and at different locations are investigated numerically at the low Reynolds number of $1.6 \times 105$. Total of three positions $(0.05 \mathrm{c}, 0.1 \mathrm{c}$ and $0.2 \mathrm{c}$ from the leading edge of airfoil), and four sizes (with heights $0.3 \mathrm{~mm}, 0.7 \mathrm{~mm}, 1 \mathrm{~mm}$ and $1.5 \mathrm{~mm}$, and constant width $4 \mathrm{~mm}$ ) of rectangular BCPs are simulated in ANSYS Fluent software using Transition SST model. The results indicate that the rectangular cross-section burst control plate is an effective device in the suppression of airfoil stall. For $0.7 \mathrm{~mm}$ and $1 \mathrm{~mm}$ height BCPs, the stall angle is postponed by $2^{\circ}$ for all positions, while for $0.3 \mathrm{~mm}$ and $1.5 \mathrm{~mm}$ height BCPs, the reduction in sudden fall of lift can be observed but at the cost of reduction in maximum lift coefficient. Among various configurations, the $1 \mathrm{~mm}$ height BCP located at $0.2 \mathrm{c}$ position is found to be most effective in the suppression of stall.
\end{abstract}

Keywords: Airfoil, Burst control plate, Laminar separation bubble, Low Reynolds number flows

\section{Introduction}

When an airfoil is introduced into the low Reynolds number flight regime (i.e. takeoff and landing, unmanned and micro air vehicles, wind turbines, sailplanes, and reduced-scale testing), the effects of viscosity are stronger which suppresses flow and boundary layer instabilities, thus enabling an smooth boundary layer that is extremely susceptible to separation. [1]

Reynolds number (Re), which is the ratio of inertia forces over viscous forces, is considered to be low if $\operatorname{Re}<5 \times 10^{5}$. [2] At low Re, performance of an airfoil degrades because of the higher drag and lower 'maximum lift' values. Hence, the flow in the boundary layer is mainly laminar, relatively fragile and thick. Consequently, gaining very high maximum lift at low Re can be challenging. [3]

The performance of almost all model aircrafts is strongly dependent on laminar separation bubbles (LSB) induced on the upper surface of the airfoil, which may occur at low Reynolds number. Some of the known conditions for the formation of an LSB are functions of Reynolds number of the flow, pressure distribution, airfoil curvature, surface roughness, and turbulence intensity of the free-stream. In the case of low Re airfoils, the flow is initially laminar and attached over the airfoil. Low energy in such flows creates adverse pressure gradient, which makes the laminar boundary layer to separate from the airfoil surface, from the point of minimum pressure. Such a separation bubble is due to adverse pressure gradient. The separated flow is highly unstable resulting in transition immediately downstream, causing the flow to become turbulent. Once the turbulent shear layer gains energy sufficient to counteract the adverse pressure gradient, the separated flow reattaches to the airfoil surface as turbulent boundary layer. Thus, a dead or recirculating air zone is formed in between separation and reattachment known as laminar separation bubble. There is almost no energy exchange with the outer flow, which makes the laminar separation bubble quite stable. The laminar separation bubble is classified into a short bubble and a long bubble. With increasing angle of attack, the chord wise length of the short bubble shortens and its position is shifted toward the leading edge The short 
bubble then fails to reattach to the airfoil surface, which is known as short bubble burst and the lift coefficient decreases abruptly causing the airfoil stall. [4-5].

Many investigations on the airfoil stall suppression have been conducted in attempt to minimize the abrupt decrease in airfoil's lift characteristics after stall. Some classical devices such as leading edge slats, vortex generators, grits, wires and boundary layer trips have been developed as passive control devices to produce disturbances that could lead to the early flow transition of the boundary layer and consequently delay airfoil stall. In the recent times, active control devices and techniques such as blowing jet into the boundary layer, oscillatory actuators and synthetic jet actuators have been investigated[5, 6, 7].

Rinoieet. al. made the use of a thin plate placed on an NACA0012 airfoil, and confirmed it was effective for burst suppression of laminar separation bubbles formed on the leading-edge of an airfoil. Consequently, it increases both the stall angle and the maximum lift coefficient at a chord Reynolds number of $1.3 \times 10^{5}$. The trailing-edge of the plate (i.e. chordwise position and height of the plate) played a crucial role in determining the performance of the plate. Grager, et. al. conducted research using a "dynamic burst control plate" which dynamically oscillates the plate. They indicated effectiveness of this type of burst control plate for stall suppression. Another study was conducted preliminarily by Kurita, et. al. It showed that the application of the rectangular cross section burst control plate was also effective for stall suppression (see Figure 1a).

With well-developed modern CFD methods and computational power, turbulent and transitional flow can be modeled effectively and accurately. Application of numerical simulation can provide deep insight into the flow physics and other aerodynamic effect on the performance of airfoil. Due to limited computational requirements, Reynolds Averaged Navier-Stokes (RANS) methods are efficient and feasible CFD simulation tool for analyzing complex flow phenomena. However, widely used RANS models such as k-omega, k-epsilon, etc assume a fully turbulent regime in entire flow field [8].

Based on these arguments, the effectiveness of rectangular cross section burst control plate (BCP) of various sizes and at various locations has been investigated performing two-dimensional numerical simulations using ANSYS Fluent 15.0, in this paper. The rectangular cross section burst control plate used in the present work has a constant width, w, along the wing span direction for all heights and positions. As illustrated in Figure 1(b), the location of the plate was defined by the chordwise distance (indicated as $\mathrm{x}_{\mathrm{p}}$ ) between the leading-edge of the airfoil and the plate. Please note $\mathrm{x}_{\mathrm{p}}$ was defined as the distance measured along the airfoil chord line $\mathrm{x}_{\mathrm{c}}$. The height of the plate is indicated as $\mathrm{h}$.
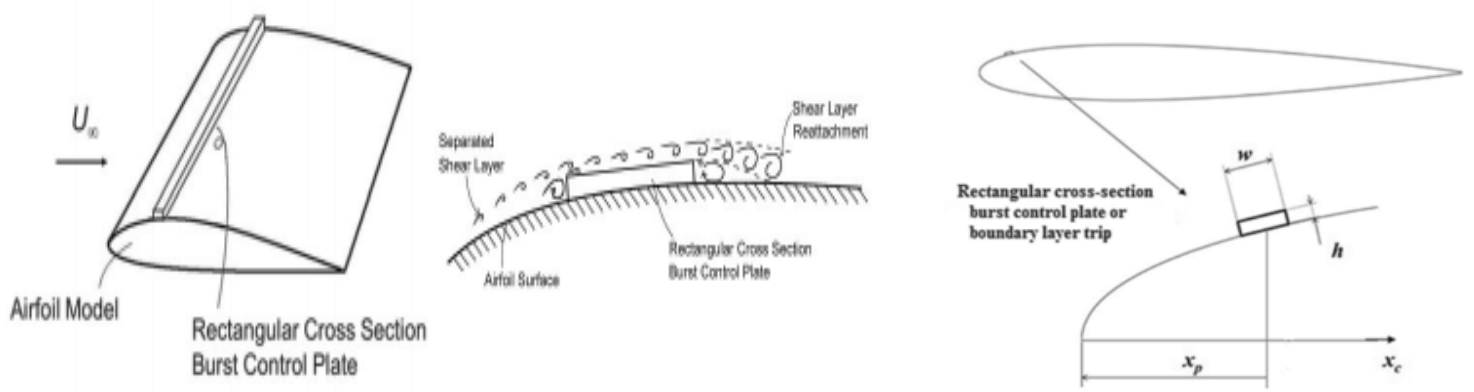

Figure 1(a):Airfoil with rectangular cross-section burst control plate
Figure 1(b):Definition of rectangular burst control plate 
2. Computational Method

One single turbulence model cannot be used as an ultimate solution for all simulations. The flow field around the airfoil is obtained by numerically solving the two-dimensional Reynolds-Averaged NavierStokes (RANS) equations. A numerical analysis has been carried out using four turbulence models: one-equation S-A model, Realizable $k-\varepsilon$ model, $k-\omega$ SST model and Transition SST. The lift and drag coefficients results of the simulations of NACA0015 airfoil at Reynolds number of $1.6 \times 10^{5}$ are compared with the experimental work carried out by Suvanjumrat, 2016 to determine which one to be chosen in present study.

\subsubsection{Computational Mesh, Boundary Conditions and Solver Setup}

Among various kinds of domains which one can use for simulating the flow over an airfoil, a C-type domain is used in this investigation because of its simplicity and convenience in creating block mesh, and is ideal for 2D airfoils with a sharp or blunt trailing edge. The airfoil has $200 \mathrm{~mm}$ chord (c) length. In order to eliminate far field boundary influence or/and to improve the stability of the numerical simulations, the outer boundary is located far away from the airfoil. Therefore, the present computational domain is a $\mathrm{C}$-type domain which has radius $\mathrm{R}=15 \mathrm{c}$ and downstream length $\mathrm{L}=30 \mathrm{c}$ as shown in Figure 2(a).

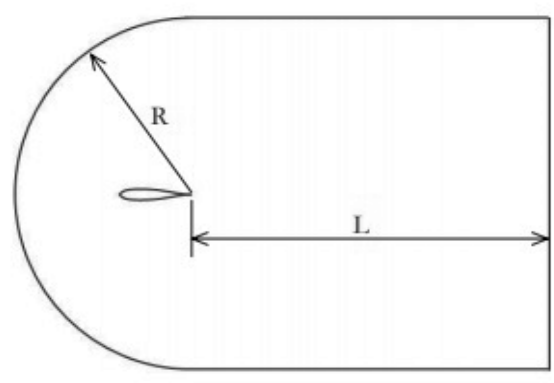

Figure 2(a):The C-type domain

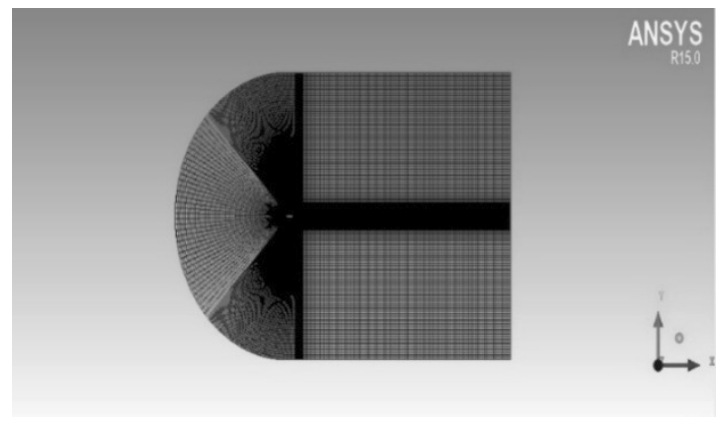

Figure 2(b):Far view of structured grid

2D airfoil points are imported into CatiaV5R20 and a surface is generated. This model is later imported into the ICEM module available in the ANSYS package for meshing. Structural quadratic Ctype grid as shown in Figures 2(b), 2(c) and 2(d) is constructed by the preprocessor ANSYSICEMCFD 15.0 software. A denser mesh is used in the regions of interest such as the boundary layer and the airfoil near-wake regions. The grid is finer around the airfoil to ensure $\mathrm{y}^{+}$value, which is a non-dimensional distance from the wall to the first node of the grid, below unity for precise simulation of the boundary layer flows. The boundary conditions used during all simulations are as follow: on the airfoil, no slip conditions are used for the velocity and Neumann boundary conditions for the pressure. On the right of the numerical domain, outflow conditions are imposed, which are Neumann boundary condition for the velocity and zero pressure. On the rest of the domain boundaries, inflow conditions are considered $\left(\mathrm{V}=\mathrm{V}_{\infty}\right)$ and Neumann boundary condition for the pressure, where $\mathrm{V}_{\infty}$ is the freestream velocity. 

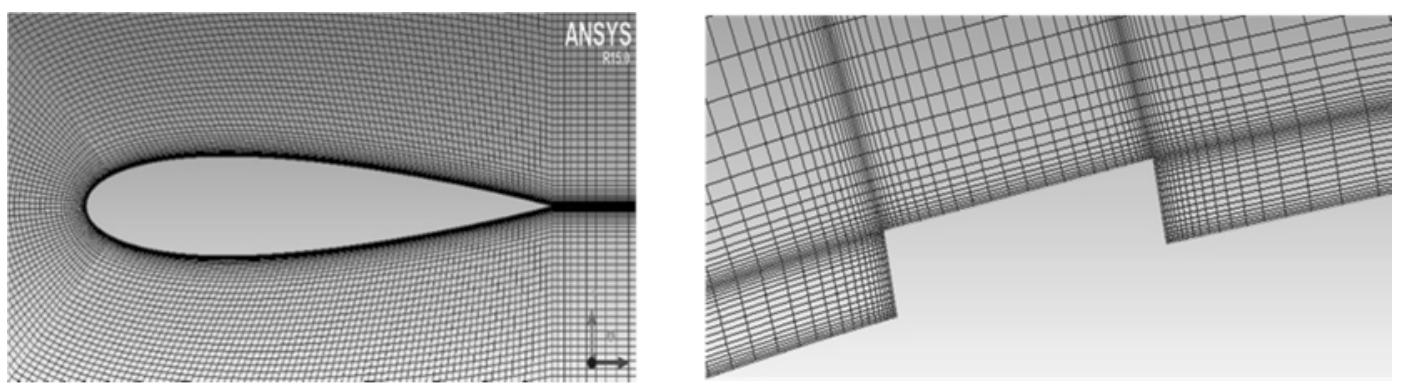

figure 2(c): Close view of dense grid figure 2(d): Grid near rectangular BCP

The commercial software ANSYS Fluent 15.0, which is based on finite volume method, is used in the computation. The solver is set for steady state. The flow is incompressible, hence a pressure-based approach is used. The input parameters such as pressure, density and viscosity are considered at sea level conditions. The turbulence intensity is set at $1 \%$. Velocity at inlet is specified to achieve the desired Re. In order to avoid calculation errors, double precision is set. To solve the coupled problem between pressure in momentum equations and velocity components, semi-implicit method for pressure-linked equations (SIMPLE) algorithm is employed, and second-order upwind spatial discretization is set in calculation. The spatial gradient is selected as the least squares cell based. Convergence criteria residual target values were set to $10^{-6}$.

\subsubsection{Grid Independence Study}

Different sized grids such as 20,825 (A), 43,450 (B), 87,310 (C), 118,455 (D), 163,155 (E) for $\operatorname{Re}=$ $1.6 \times 10^{5}$ at $\alpha=8^{\circ}$ were compared to ensure grid independence of the calculations and finally the 87,310 grids was chosen since difference of the results of lift coefficient $\left(\mathrm{C}_{\mathrm{L}}\right)$ values were negligible after this grid size as shown Figure 2(e).

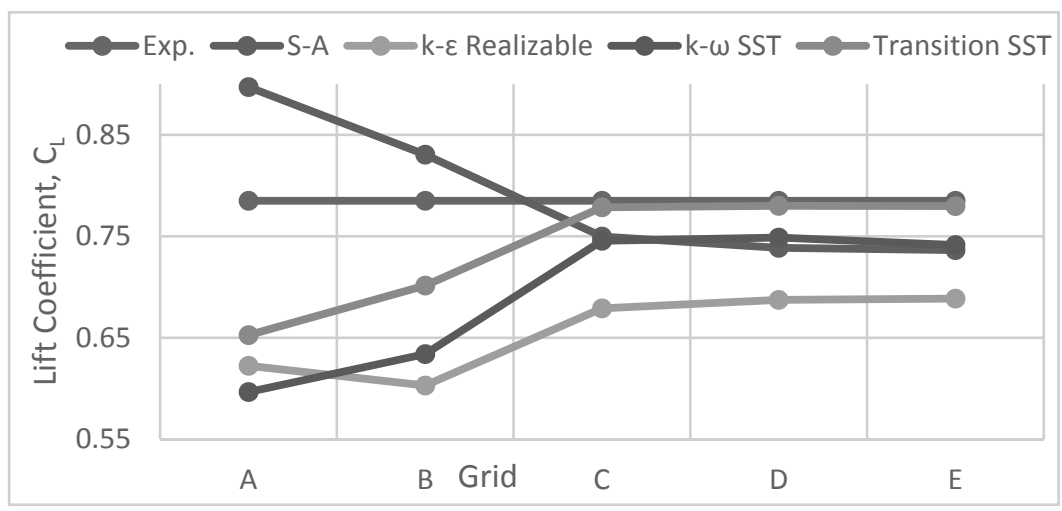

Figure 2(e): Grid independence study of lift coefficient, $C_{L}$ versus grid sizeat $\alpha=8^{\circ}$

Transition SST model gives the best solution when compared with S-A, Realizable k- $\varepsilon$ and k- $\omega$ SST models because the line is closer to the experimental data line.

\subsection{Model Validation}

Extensive simulations were carried out on a clean NACA 0015 airfoil using four turbulence models, namely the Transition SST, k- $\omega$ SST, realizable k- $\varepsilon$ and S-A model based on same cell structure domain. In Figures 2(f), $2(\mathrm{~g})$ and $2(\mathrm{~h})$, computed lift coefficients $\left(\mathrm{C}_{\mathrm{L}}\right)$, drag coefficients $\left(\mathrm{C}_{\mathrm{D}}\right)$ and sliding ratio $\left(\mathrm{C}_{\mathrm{L}} / \mathrm{C}_{\mathrm{D}}\right)$ values varying angles of attack (AOA, $\left.\alpha\right)$ from $0^{\circ}$ to $20^{\circ}$ with an interval of $2^{\circ}$ at Reynolds number of $1.6 \times 10^{5}$ are compared with the experimental data of Suvanjumrat, 2016 [9]. 
The simulation results of all turbulence models are in good agreement with the experimental data especially in the pre-stall region. Numerical results do not exhibit completely satisfactory results in the stall region due to RANS modeling weakness for stalled condition where the flow is highly unsteady. The Transition SST and k- $\omega$ SST models give almost similar results for $C_{\mathrm{L}}$ values (except for angles between $10^{\circ}$ and $16^{\circ}$ ) because they hold similar transport equations for $\mathrm{k}$ and $\omega$ to solve the flow, while S-A model has only one transport equation to model the kinematic viscosity but can solve the flow quicker. The S-A and $k-\varepsilon$ Realizable models' prediction of $\mathrm{C}_{\mathrm{L}}$ and $\mathrm{C}_{\mathrm{D}}$ valuesdeviates significantly from experimental data when the angles of attack are higher than $10^{\circ}$.

It is obvious that the Transition SST model yields the smallest discrepancy among the four models. For example, it is able to capture the peak lift of 0.8293 at $\alpha=10^{\circ}$. The $C_{L}$ curve follows a linear pattern up to this value. It indicates that the flow remains attached up to this maximum value and the drop in value thereafter indicates separated flow or stall. In addition, the predicted drag coefficients by the Transition SST model also agree very well with the experimental data. The Transition SST model predicts the peak value of sliding ratio, 38 at $\alpha=8^{\circ}$, which is a good match with the experimental peak value of 40 at the same angle of attack. Average deviation of 10\% in lift prediction and $8 \%$ in drag prediction is observed between simulation for Transition SST model and experimental results, and fall in the acceptable range. The interaction of different numerical schemes and mesh density may lead to error.

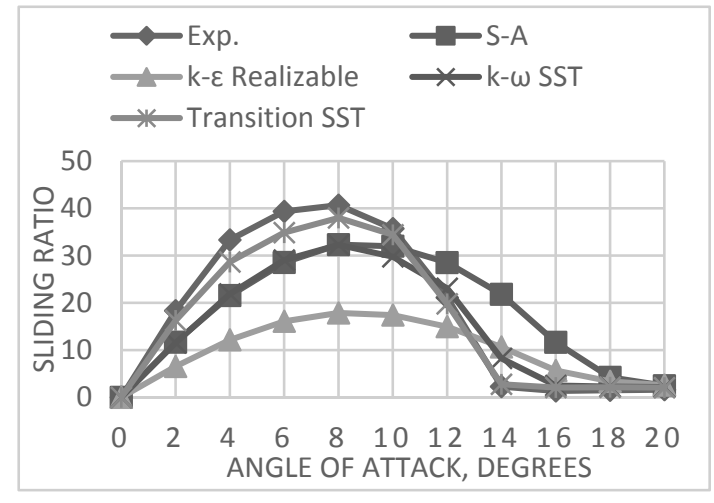

Figure 2(f): Lift coefficient Vs AOA curve

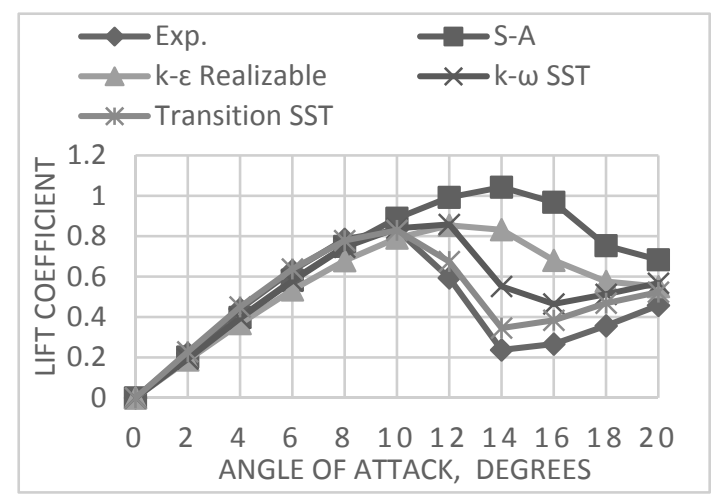

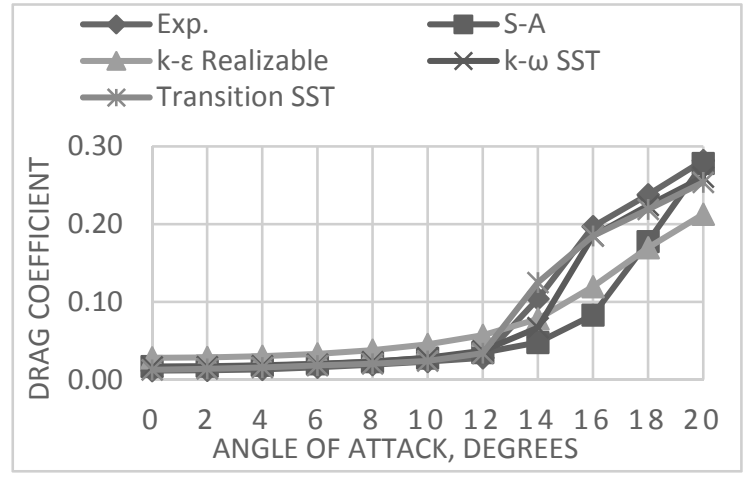

Figure 2(g): Drag coefficient VsAOA curve

Figure 2(h): Sliding Ratio VsAOA curve

The coefficients of pressure, $\mathrm{C}_{\mathrm{P}}$ plots signify a much better comparison of numerical and experimental results. Figure 2(i)presents a comparison between the pressure coefficients of the current work and the experimental measurements of Miller, 2008 [10] for the NACA 0015 airfoil at Reynolds number of $2.4 \times 10^{5}$ and angles of attack of $0^{\circ}$ and $10^{\circ}$. 


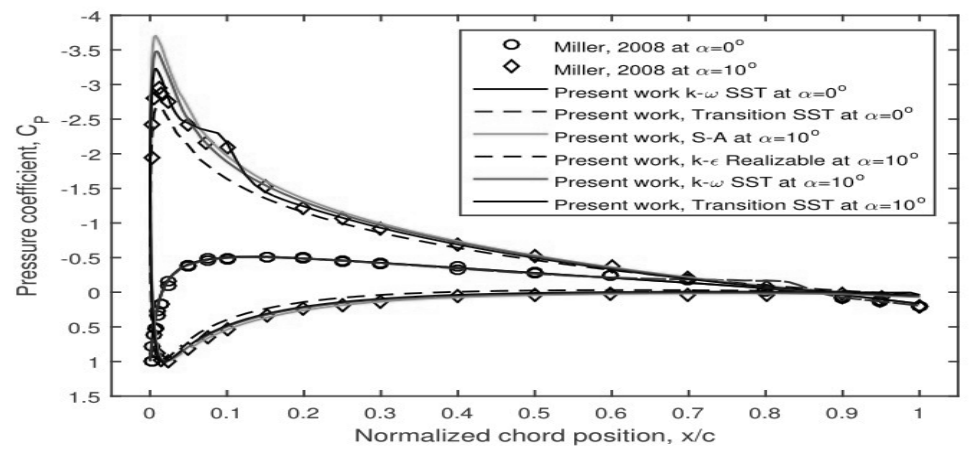

Figure 2(i): Comparison of pressure coefficient distribution of different turbulence models

The Cp plot for the Transition SST model is seen to capture the transition effects i.e. the formation of LSB represented by a hump in the plot, and agree well with the experimental $C_{P}$ plot, which indicates that using Transition SST model for further simulations could obtain reasonable and accurate results. This behavior is not captured by the S-A, $k-\varepsilon$ Realizable and k- $\omega$ SST turbulence models. Hence, it can be inferred that S-A, $k-\varepsilon$ Realizable and k- $\omega$ SST are good turbulence models, very useful in case of flows where LSB does not exist. Also, the simulation results obtained for lift and drag coefficients using Transition SST model will be used as baseline study (clean airfoil).

\section{Results and Discussion}

To investigate the effects of plate position over flow around the airfoil, computations were made using the rectangular cross-section BCP of various sizes and at various locations. Four BCPs of 0.3 $\mathrm{mm}, 0.7 \mathrm{~mm}, 1 \mathrm{~mm}, 1.5 \mathrm{~mm}$ height and $4 \mathrm{~mm}$ width were tested at $0.05 \mathrm{c}, 0.1 \mathrm{c}$ and $0.2 \mathrm{c}$ from the leading edge of the airfoil, where $\mathrm{c}=$ chord.

Figures 3(a) and 3(b) shows surface pressure distributions of a clean airfoil and of an airfoil with BCP height of $1 \mathrm{~mm}$ located at $0.2 \mathrm{c}$. The figure indicates that a short bubble was formed for the angle of attack, $\alpha=10^{\circ}$ with the clean airfoil (see the high section pressure near the leading edge followed by a plateau area and a sudden pressure recovery which indicates flow reattachment). Both high suction pressure near the leading edge and sudden pressure recovery were totally lost when $\alpha$ was increased to $14^{\circ}$ for this airfoil, which means the airfoil has stalled. For the case of airfoil with BCP, the high suction pressure peak was observed at $\alpha=12^{\circ}$, which means the stall was suppressed at least until $\alpha$ $=12^{\circ}$.
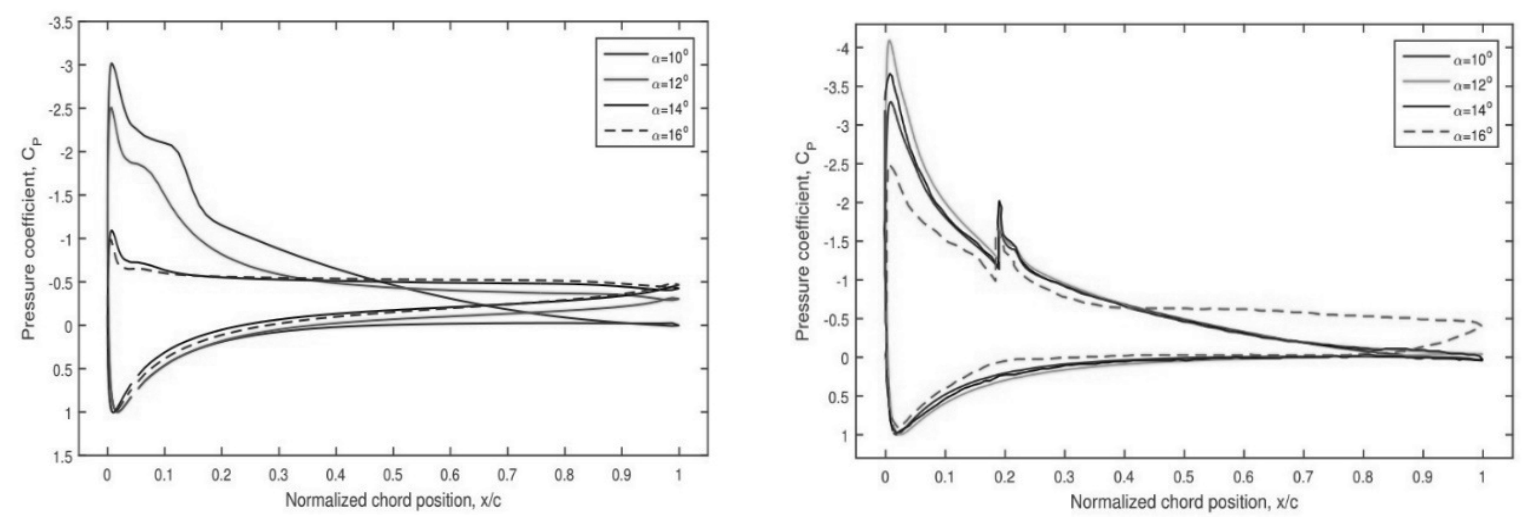

Figure 3: $\mathrm{C}_{\mathrm{P}}$ distributions with variations of $\alpha($ a) Clean airfoil (b) $1 \mathrm{~mm}$ height $\mathrm{BCP}$ at $0.2 \mathrm{c}$ location

Figures 3(c), 3(e), 3(g) and 3(i) show lift coefficients of BCP height $0.3 \mathrm{~mm}, 0.7 \mathrm{~mm}, 1 \mathrm{~mm}$ and 1.5 $\mathrm{mm}$ located at $0.05 \mathrm{c}, 0.1 \mathrm{c}$ and $0.2 \mathrm{c}$ and compared to the clean airfoil (baseline). The results indicate that $\mathrm{BCP}$ of all heights for all positions gives lower $\mathrm{C}_{\mathrm{L}}$ when compared to the clean airfoil for angles 
of attack, less than $10^{\circ}$. When the angles are between $10^{\circ}$ and $16^{\circ}$, the lift coefficients for BCPs of height $0.7 \mathrm{~mm}$ and $1 \mathrm{~mm}$ are significantly higher than the value of the baseline airfoil for all positions, while for BCPs with height $0.3 \mathrm{~mm}$ and $1.5 \mathrm{~mm}$, the stalling effect has become somewhat less severe in between the same range of angle of attack because of the reduced efficiency in the reattachment of the separated boundary layer. In particular, the maximum lift coefficients at maximum stall angle $\alpha=12^{\circ}$ for BCPs of height $0.7 \mathrm{~mm}$ and $1 \mathrm{~mm}$ at the location $0.2 \mathrm{c}$ are 0.8788 and 0.897 , which is over $6 \%$ and $8 \%$ higher than the maximum lift value $\left(\alpha=10^{\circ}\right)$ of clean airfoil. It should be noted that the stall angle difference between clean airfoil and airfoils with BCPs of height $0.7 \mathrm{~mm}$ and $1 \mathrm{~mm}$ for all positions is $2^{\circ}$. In case of BCP with $0.3 \mathrm{~mm}$ height at the location $0.2 \mathrm{c}$, the lift coefficient at $\alpha=10^{\circ}$ is 0.8476 , which is $2 \%$ higher when compared to the maximum lift value of the clean airfoil. And, in case of BCP with height $1.5 \mathrm{~mm}$ at the location $0.2 \mathrm{c}$, the maximum value of lift is at $\alpha=10^{\circ}$, which is $6.5 \%$ less than the maximum lift value of clean airfoil. It is found that for each BCP height, the location that generated the highest lift is at $0.2 \mathrm{c}$. This may be because the size of LSB is reduced and the separated flow reattaches downstream of the flow due to reenergized separated boundary layer.

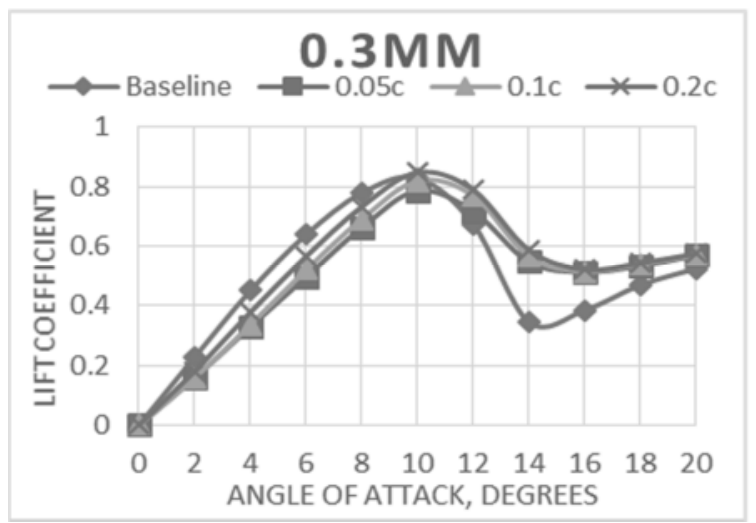

Figure 3(c):Lift coefficient VsAOA

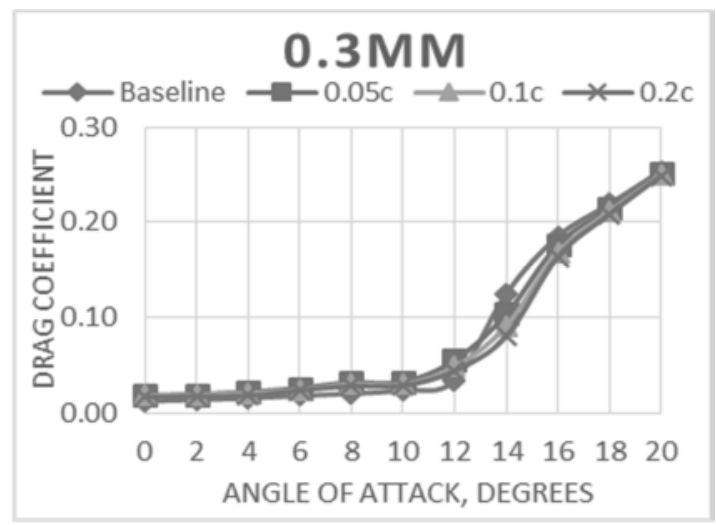

Figure 3(d): Drag coefficient VsAOA

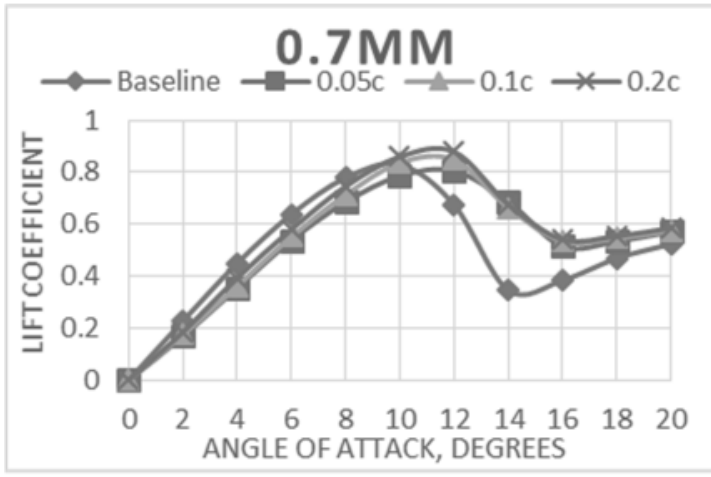

Figure 3(e):Lift coefficient VsAOA

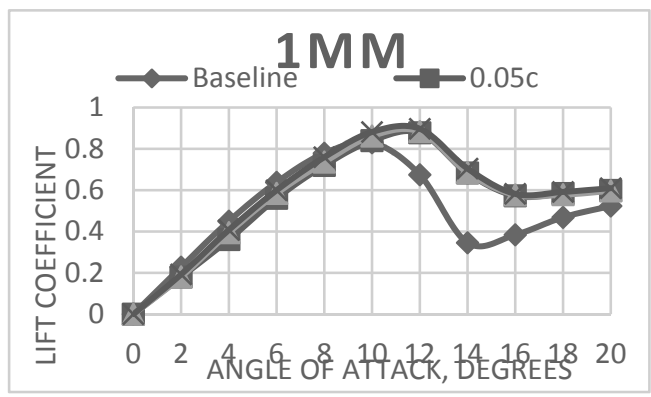

Figure 3(g):Lift coefficient Vs AOA

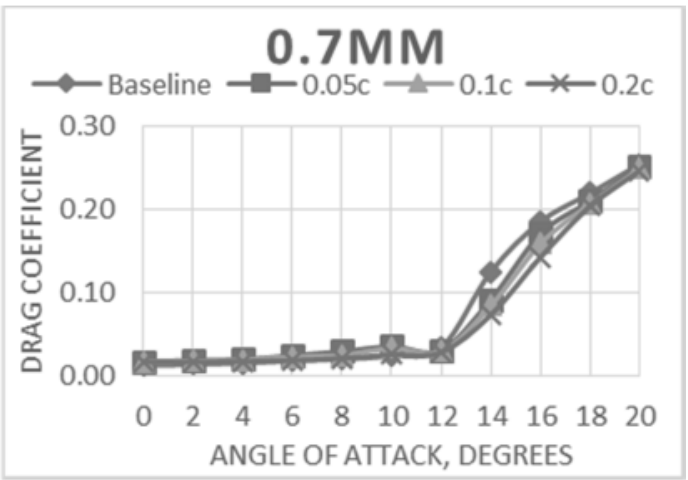

Figure 3(f): Drag coefficient VsAOA

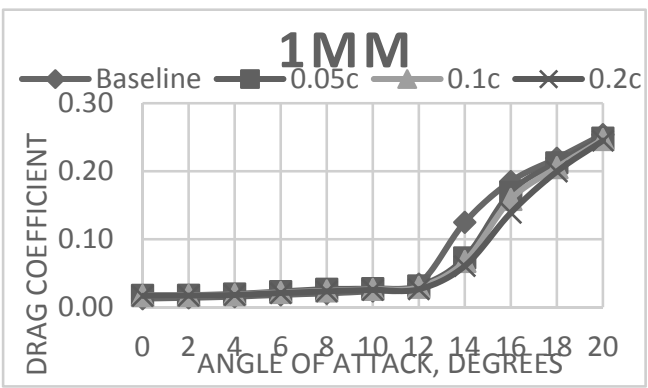

Figure 3(h): Drag coefficient VsAOA 


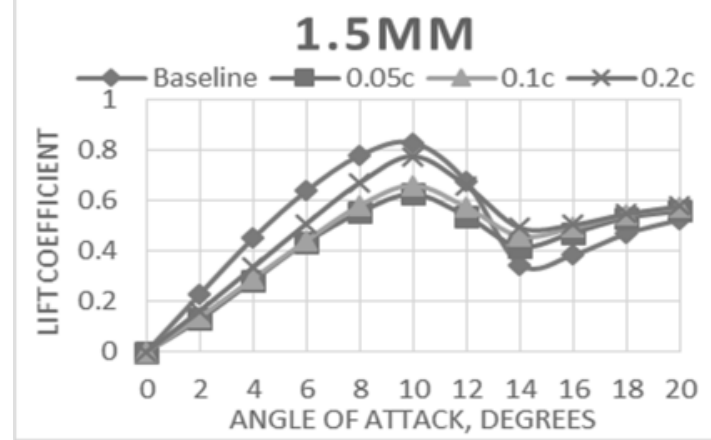

Figure 3(i):Lift coefficient Vs AOA

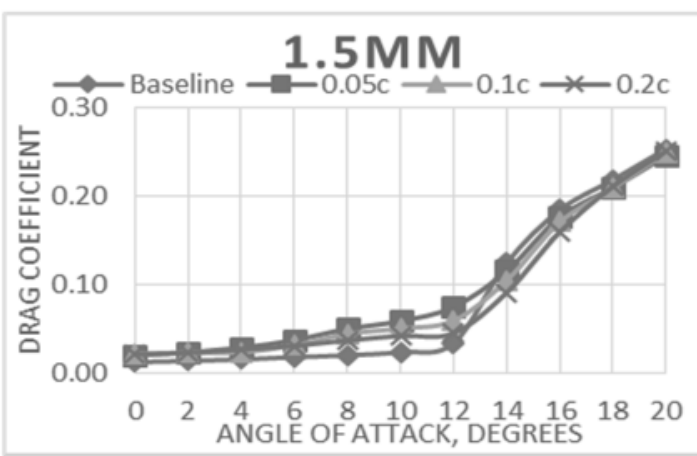

Figure 3(j): Drag coefficient VsAOA

Figures 3(d), 3(f), 3(h) and 3(j) show drag coefficients of BCP height of $0.3 \mathrm{~mm}, 0.7 \mathrm{~mm}, 1 \mathrm{~mm}$ and $1.5 \mathrm{~mm}$ located at $0.05 \mathrm{c}, 0.1 \mathrm{c}$ and $0.2 \mathrm{c}$ and compared to the baseline data. The results indicate that BCPs of all heights for all positions give more $C_{D}$ when compared to the clean airfoil for angles of attack less than $10^{\circ}$. The drag increase comes from the skin friction due to the transition region that exists on suction surface of airfoil. The BCP on the surface of the airfoil increases the surface roughness in the flow field near the leading edge of the airfoil. When the angles of attack are between $10^{\circ}$ and $16^{\circ}$, drag is successfully suppressed for BCPs with heights $0.7 \mathrm{~mm}$ and $1 \mathrm{~mm}$ for all positions. In particular, the drag coefficient for $\mathrm{BCP}$ with height $1 \mathrm{~mm}$ at $0.2 \mathrm{c}$ position is significantly lower than the values for other cases with the maximum drag reduction of over $50 \%$ at $\alpha=14^{\circ}$ when compared to baseline data. The performance of the airfoil (L/D) is greatly increased between these angles of attack. On the other hand, the drag coefficient increases gradually due to turbulent boundary-layer separation at the rear portion of the airfoil moving upstream with increasing angle of attack. When the angle of attack is greater than $18^{\circ}$, the drag coefficients are almost same, i.e. seem to converge with the case clean airfoil for all configurations. Also, it can be observed that for each BCP height, the location which generated the lowest $C_{D}$ is at $0.2 \mathrm{c}$.Figure $3(\mathrm{k})$ show lift coefficients of $\mathrm{BCP}$ heights of $0.3 \mathrm{~mm}, 0.7 \mathrm{~mm}, 1 \mathrm{~mm}$ and $1.5 \mathrm{~mm}$ located at $0.2 \mathrm{c}$ from the leading edge and compared to the airfoil without $\mathrm{BCP}$. The results indicate that $1.5 \mathrm{~mm}$ height $\mathrm{BCP}$ produces the lowest $\mathrm{C}_{\mathrm{L}}$ for angles of attack between $0^{\circ}$ and $12^{\circ}$. When the angle of attack is higher than $12^{\circ}$, the lift coefficients in all cases are higher than that of the airfoil with no BCP. If the BCP height is less than $1.5 \mathrm{~mm}$, the stalling effects are well-controlled because of the transformation of laminar separation bubble to turbulent boundary layer, only to get reattached to the airfoil surface with a smaller size LSB. This also assists in generating more lift when the angle of attack is $10^{\circ}$ or greater for BCPs with height 0.3 $\mathrm{mm}, 0.7 \mathrm{~mm}$ and $1 \mathrm{~mm}$. The $1 \mathrm{~mm}$ height BCP generates the maximum lift in that range, and was the most effective one in suppressing airfoil stall.

Figure 3(1) shows drag coefficients of BCPs with height $0.3 \mathrm{~mm}, 0.7 \mathrm{~mm}, 1 \mathrm{~mm}$ and $1.5 \mathrm{~mm}$ located at the position $0.2 \mathrm{c}$ and compared to the clean airfoil. The results indicate that $1 \mathrm{~mm}$ height $\mathrm{BCP}$ generates the lowest $C_{D}$ for almost all angles of attack when compared to other configurations.

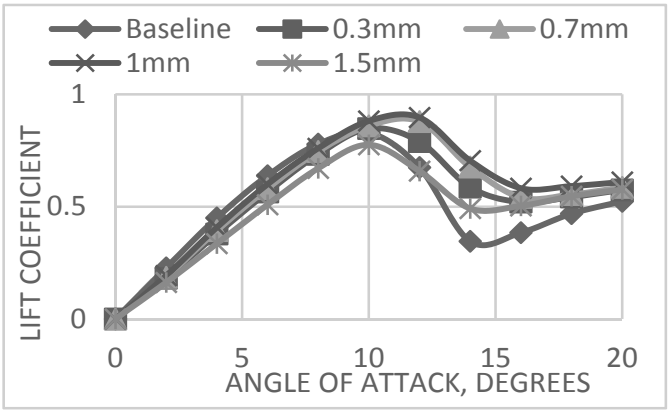

Figure 3(k): Lift coefficient vsAOA

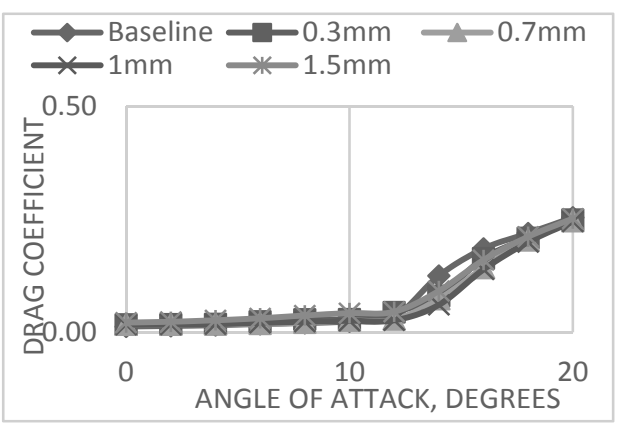

Figure 3(1) Drag coefficient vsAOA 
An advantage of BCP is that it makes the lift coefficient after stalling to reduce more smoothly. The effects of addition of BCPs to airfoil's surface are dependent on location and size. From above results, it can be inferred that the $1 \mathrm{~mm}$ height $\mathrm{BCP}$ located at $0.2 \mathrm{c}$ has the most advantage in lift enhancement and drag reduction when compared to the BCPs located at other positions.

\section{Conclusion}

In this research, for the purpose of airfoil stall suppression BCPs of four different sizes (with heights $0.3 \mathrm{~mm}, 0.7 \mathrm{~mm}, 1 \mathrm{~mm}$ and $1.5 \mathrm{~mm}$, and constant width $4 \mathrm{~mm}$ ) added on NACA 0015 airfoil surface were simulated at three different locations $(0.05 \mathrm{c}, 0.1 \mathrm{c}$ and $0.2 \mathrm{c}$ from the leading edge of airfoil) at the low Re of $1.6 \times 10^{5}$ airfoil using ANSYS Fluent software. The results led to the conclusion that the addition of BCPs as a method of controlling stall characteristics is successful with an improved airfoil performance. $1 \mathrm{~mm}$ height rectangular $\mathrm{BCP}$ was found to be the optimum size at the optimum location of $0.2 \mathrm{c}$ from the leading edge of airfoil for the control of stall. $1.5 \mathrm{~mm}$ height BCP for all positions was seen to control the sudden fall of reduction of lift after stall angle, however at the sacrifice of maximum lift coefficient value. The findings from this work may contribute in future design and development of burst control plates.

\section{References}

1. Patrick, M. T. (2011). "Investigation of a Laminar Airfoil with Flow Control and the Effect of Reynolds Number". The Ohio State University: Department of Aeronautical and Astronautical Engineering.

2. Shah, H., Bhattarai, N., Mathew, S., \& Lim, C. M. (2012). "Low Reynolds number airfoil for small horizontal axis wind turbine blades". Sustainable future energy 2012 and 10th SEE FORUM. Brunei Darussalam.

3. Giguere, P., \& Selig, M. S. (1997)."Low Reynolds number airfoils for small horizontal axis wind turbines". Wind Engineering.

4. Kurita, S., Rinoie, K., \& Sunada, Y. (Jun. 2008). "Stall Suppression by Use of a Rectangular Cross Section Plate Placed on NACA 0012 Airfoil". (pp. 331-334). Sendai, Japan: Proc. the 40th Fluid Dynamics Conference.

5. Rinioe, K., Okuno, M., \& Sunada, Y. (2009). "Airfoil Stall Suppression by Use of a Bubble Burst Control Plate". AIAA Journal, 47 (2): 322-330.

6. Grager, T., Rothmayer, A., \& Hu, H. (2011). "Stall Suppression of a Low-Reynolds-Number Airfoil with a Dynamic Burst Control".AIAA Paper 2011-1180.

7. Lyon, C. A., Selig, M. S., \& Broeren, A. P. (1997). "Boundary Layer Trips on Airfoils at Low Reynolds Numbers". AIAA Paper, 35.

8. ANSYS Fluent 15.0 Theory Guide. (2015).

9. Suvanjumrat, C. (2016, October 17). "Comparison of Turbulence Models for Flow Past NACA0015 Airfoil Using OpenFOAM". Engineering Journal, 21(3). Retrieved from http://www.engj.org/

10. Miller, D. S. (2008). Lift, Drag and Moment of NACA 0015 Airfoil. OHIO State University, Department of Aerospace Engineering. 